This item was submitted to Loughborough's Research Repository by the author.

Items in Figshare are protected by copyright, with all rights reserved, unless otherwise indicated.

\title{
Polyvinyl butyral composites containing halloysite nanotubes/reduced graphene oxide with high dielectric constant and low loss
}

PLEASE CITE THE PUBLISHED VERSION

https://doi.org/10.1016/j.cej.2020.124910

\section{PUBLISHER}

Elsevier

\section{VERSION}

AM (Accepted Manuscript)

\section{PUBLISHER STATEMENT}

This paper was accepted for publication in the journal Chemical Engineering Journal and the definitive published version is available at https://doi.org/10.1016/j.cej.2020.124910.

\section{LICENCE}

CC BY-NC-ND 4.0

\section{REPOSITORY RECORD}

Su, Yaotian, Mingping Zhou, Gang Sui, Jinle Lan, Hongtao Zhang, and Xiaoping Yang. 2020. "Polyvinyl Butyral Composites Containing Halloysite Nanotubes/reduced Graphene Oxide with High Dielectric Constant and Low Loss". Loughborough University. https://hdl.handle.net/2134/12078741.v1. 


\title{
Polyvinyl butyral composites containing halloysite nanotubes/reduced graphene oxide with high dielectric constant and low loss
}

Yaotian Su, ${ }^{\mathrm{a}}$ Mingping Zhou, ${ }^{\mathrm{a}}$ Gang Sui, ${ }^{\mathrm{a}}$ Jinle Lan, ${ }^{\mathrm{a}}$ Hongtao Zhang, ${ }^{\mathrm{b}}$ Xiaoping Yang ${ }^{\mathrm{a}}$

${ }^{a}$ State Key Laboratory of Organic-Inorganic Composites, College of Materials Science and Engineering,

Beijing University of Chemical Technology, Beijing, 100029, China

${ }^{\mathrm{c}}$ Department of Materials, Loughborough University, Leicestershire, UK

*Corresponding authors:

* Gang Sui. Email: suigang@ mail.buct.edu.cn

\begin{abstract}
Polymer-based composites with high dielectric constant and low loss are highly desirable due to their inherent advantages of easy processability, flexibility, and lightweight. Herein, a functional nanofillers, halloysite nanotubes (HNTs) decorated reduced graphene oxide (rGO) hybrid microstructures (HNTs@rGO) was successfully prepared via controllable electrostatic self-assembly and in-situ heat reduction method. These hybrid microstructures combine characteristics of natural 1D ceramic nanotubes with large aspect ratio and high electric conductivity of rGO micro-sheets, which provided ideal material collocation in the construction of microcapacitors. The HNTs not only effectively prevented direct contact between the rGO micro-sheets in the composites but also played an important role in forming dielectric interface within microcapacitors. Consequently, an HNTs@rGO/polyvinyl butyral (PVB) composites
\end{abstract}


containing a very low content of 5wt $\%$ rGO exhibited an ultra-high dielectric constant of 150 and an extremely low loss of 0.12 at $10^{3} \mathrm{~Hz}$. It is believed that the unique characteristics and facile fabrication process of HNTs@rGO/PVB composite make it a potentially excellent candidate for flexible polymer-based dielectric materials applied in the capacitor fields.

Keywords: Polymer-matrix composites; Dielectric properties; Halloysite nanotubes; Graphene oxide

\section{Introduction}

Flexible materials with high dielectric constant and low dielectric loss (tan $\delta)$ play an important role in the fabrication of thin-film capacitor and wearable devices in the modern electronic field [1-5]. Polymer-based composites combining intrinsic advantages from both polymer and inorganic nanoparticles possess great potential in the industrial field of dielectric materials because of their excellent flexibility, easy processability, lightweight and low cost [6]. It has been widely investigated that polymers filled with conductive nanoparticles such as metal powders, carbon nanotubes, graphenes, MXenes can achieve very high dielectric constant, but these materials are inevitably accompanied by a huge increase of dielectric loss due to the formation of conductive networks between nanoparticles, which is termed as a percolative phenomenon [7-10].

Some studies indicated that introducing ceramic or polymer coating layers onto the surface of conductive fillers can suppress the current leakage and reduce the dielectric 
loss of the materials. Shi et al. [11] fabricated reduced graphene oxide-encapsulated barium titanate (BT-RGO) hybrid filled polyvinylidene fluoride (PVDF) composites. The composites filled with 40 vol\% BT-RGO showed suppressed dielectric loss of 0.25 $\left(10^{3} \mathrm{~Hz}\right)$ compared with RGO/PVDF $(\tan \delta>10)$. However, the relatively low dielectric constant (67.5) and deterioration of mechanical properties caused by high filler loadings (40 vol\%) hindered its further application. Wan et al. [12] prepared another sort of barium titanate coated graphene (BT@TGO) nanofillers using a sol-gel method combined with the thermal treatment process. The obtained BT@TGO/PVDF materials showed a low dielectric loss of 0.16 at $10^{3} \mathrm{~Hz}$ as the filler content reached $9.5 \mathrm{wt} \%$, which implied a suppressed current leakage due to the presence of BT coating layers. However, the dielectric constant of the composites was just 35 which cannot meet the performance requirements of dielectric functional materials with high dielectric constant (>100) in the modern electronic field. Li et al. [13] synthesized polydopamine coated reduced graphene oxide nanoparticles (PDA@RGO) through self-polymerization of dopamine onto graphene oxide $(\mathrm{GO})$ and subsequent chemical reduction process. The dielectric constant of PVDF with 0.70 wt\% PDA@RGO increased to $176\left(10^{3} \mathrm{~Hz}\right)$, about 17 times of neat PVDF, and the loss tangent was suppressed to 0.337 due to reduction of the concentration and mobility of ionizable carboxylic groups affected by PDA. Unfortunately, the reduction process in this study was complicated and not environmentally friendly so that it was not appropriate for industrial production. Besides the examples illustrated above, an increasing number of strategies have been 
developed more recently to prepare high-performance dielectric materials by incorporating "core-shell structural conductive nanofillers" into polymer matrix [14-18]. One benefit of this sort of nanofillers was that the outer insulating shell can effectively prevent the current leakage caused by the formation of conductive networks which was the main source of dielectric loss, however, the disadvantage was that the non-conductive shell completely suppressed the charge mobility, prohibiting the formation of a large number of microcapacitors. For such a system, the contribution to the dielectric constant only came from the strengthened average electric field effect based on Maxwell-Wagner theory [18], so that the dielectric constant of the material will be much lower than that filled with normal conductive fillers. Besides, some of the ceramic coating layers usually presented poor compatibility with polymer matrix because of the lacking functional groups on the surface, thus the composites will suffer a deterioration of mechanical and dielectric properties caused by defects, and the overall performance was hardly as perfect as pre-designed.

Based on the above discussion, a novel dielectric composites combining merits from both halloysite nanotubes (HNTs) and reduced graphene oxide (rGO) micro-sheets was prepared in this paper. Herein, we chose PVB as the matrix because it was a soft polar polymer with many dipole groups, which had been widely used as security glass interlayer materials but rarely studied in electronic field [19], and HNTs as nanofillers to decorate rGO because it was a low-cost ceramic nanotubes which possessed good dielectric properties [20]. In this work, the HNTs@GO micro-sheets were firstly 
synthesized and then reduced to HNTs@rGO in the PVB matrix under mild heat processing. The HNTs not only prevented direct contact between the rGO micro-sheets in the composites but also acted as high dielectric ultrathin barriers, facilitating the formation of a great number of microcapacitors with large capacitance. Based on these designs, excellent dielectric property of HNTs@rGO/PVB composites with HNTs@rGO fillers (containing 5wt $\%$ rGO) was achieved $\left(\varepsilon^{\prime}=150, \tan \delta=0.12,10^{3} \mathrm{~Hz}\right)$, which was superior to that of ceramic or polymer-coated conductive nanoparticles filled composite reported in previous research. Meanwhile, the PVB matrix provided the functional composites with desired processing performance and flexibility, which was important to the application of dielectric functional materials. The mechanism of the synergistic effect of rGO and HNTs was also investigated in this paper.

\section{Experimental}

\subsection{Materials}

Polyvinyl butyral (PVB) $(\mathrm{Mw}=50000-80000)$ was purchased from Chang Li Products \& Chemical Co., China. Halloysite clay nanotubes (HNTs, average outer diameter: $\sim 40 \mathrm{~nm}$, length: $200 \mathrm{~nm}-1 \mu \mathrm{m}$ ) were obtained from Yanbo Mineral Processing Co., China. Graphene oxide (GO, average sheet size is $1 \sim 2 \mu \mathrm{m}$ ) was produced by Xianfeng Nanomaterials Tech. Co., China. $\gamma$-aminopropyl triethoxysilane (APTES, 98\%) was purchased from Sigma-Aldrich., USA. Ethanol, Toluene and concentrated hydrochloric acid were obtained from Beijing Chemical Factory, China. The HNT powders were purified by repeated sedimentation process before use. All solvents were 
analytically pure and used as received.

\subsection{Synthesis of APTES-HNTs}

The preparation of APTES grafted HNTs (APTES-HNTs) was carried out according to the previous report. Typically, $3.6 \mathrm{~g}$ HNTs were firstly dissolved in $150 \mathrm{ml}$ dry toluene solvent and then ultrasonicated for $30 \mathrm{~min}$. After that, a certain amount of APTES was added in the mixture and ultrasonicated for another $10 \mathrm{~min}$. The mixture was then refluxed at $120{ }^{\circ} \mathrm{C}$ for $24 \mathrm{~h}$ under magnetic stirring. The product was filtered and then washed with toluene and ethanol several times to remove excess organosilane.

\subsection{Synthesis of HNTs@GO micro-sheet}

An electrostatic self-assembly method was operated to synthesize the HNTs@GO micro-sheet. APTES-HNTs were re-dispersed in the deionized water and then the $\mathrm{pH}$ value was adjusted to 1 2 to make zeta potential of solventized APTES-HNTs particles greater than zero. A GO aqueous suspension was slowly added to the APTES-HNTs mixture and brown precipitate appeared gradually, and then the suspension became transparent which indicated the HNTs@GO had been successfully synthesized. The HNTs@GO micro-sheets with different HNTs and GO weight ratios were prepared by controlling the concentration of HNTs and GO aqueous solution. The resultant hybrid material was coded as $\mathrm{x} \% \mathrm{HNTs} @ \mathrm{y} \% \mathrm{GO}$, where $\mathrm{x}$ and $\mathrm{y}$ were the weight fraction of HNTs and GO, respectively. Three different kinds of HNTs@GO micro-sheets were prepared $(\mathrm{x} / \mathrm{y}=1: 1,2: 1,3: 1)$, and the weight ratio $\mathrm{x} / \mathrm{y}=2: 1$ was used as the optimized material composition to fabricate the micro-sheets. The mechanism of the procedure 
was shown in Fig. 1.

\subsection{Preparation of HNTs@rGO/PVB composite}

For the fabrication of HNTs@rGO/PVB nanocomposite (Fig. 1), the brown precipitate was re-dissolved in ethanol and a certain amount of PVB powder was added under vigorous stirring. To make a better dispersion of HNTs@GO particles, the HNTs@GO/PVB mixture was poured into deionized water to perform an antisolvent precipitation process. The product was finally filtrated using a $0.22 \mu \mathrm{m}$ PTFE membrane and dried at $80^{\circ} \mathrm{C}$ for $3 \mathrm{~h}$. Subsequently, the mixed powder was hot-pressed at an optimized condition of $180^{\circ} \mathrm{C}$ for $2 \mathrm{~h}$ to make the GO convert to $\mathrm{rGO}$ through a heat reduction process with the removal of oxygen-containing groups [21-22]. The hot-pressing parameters were identified with the assistance of the Raman spectrum which was shown in the supplementary information (Fig S1.). The thickness of the film was approximately $0.2 \mathrm{~mm}$ (see Fig. 1 digital photos). The control samples $\mathrm{x} \% \mathrm{HNTs}+\mathrm{y} \% \mathrm{rGO} / \mathrm{PVB}$ ( $\mathrm{x}$ and $\mathrm{y}$ were weight fraction of HNTs and GO, $\mathrm{x} / \mathrm{y}=1: 1,2: 1$, 3:1), $\mathrm{y} \% \mathrm{rGO} / \mathrm{PVB}$ and $\mathrm{x} \% \mathrm{HNTs} / \mathrm{PVB}$ composites were also prepared via the antisolvent precipitation process illustrated above, and the optimized proportion was $\mathrm{x} / \mathrm{y}=2: 1$ for $\mathrm{x} \% \mathrm{HNTs}+\mathrm{y} \% \mathrm{rGO} / \mathrm{PVB}$. The pure HNT's pellet was also fabricated by cold pressing under $30 \mathrm{MPa}$. 


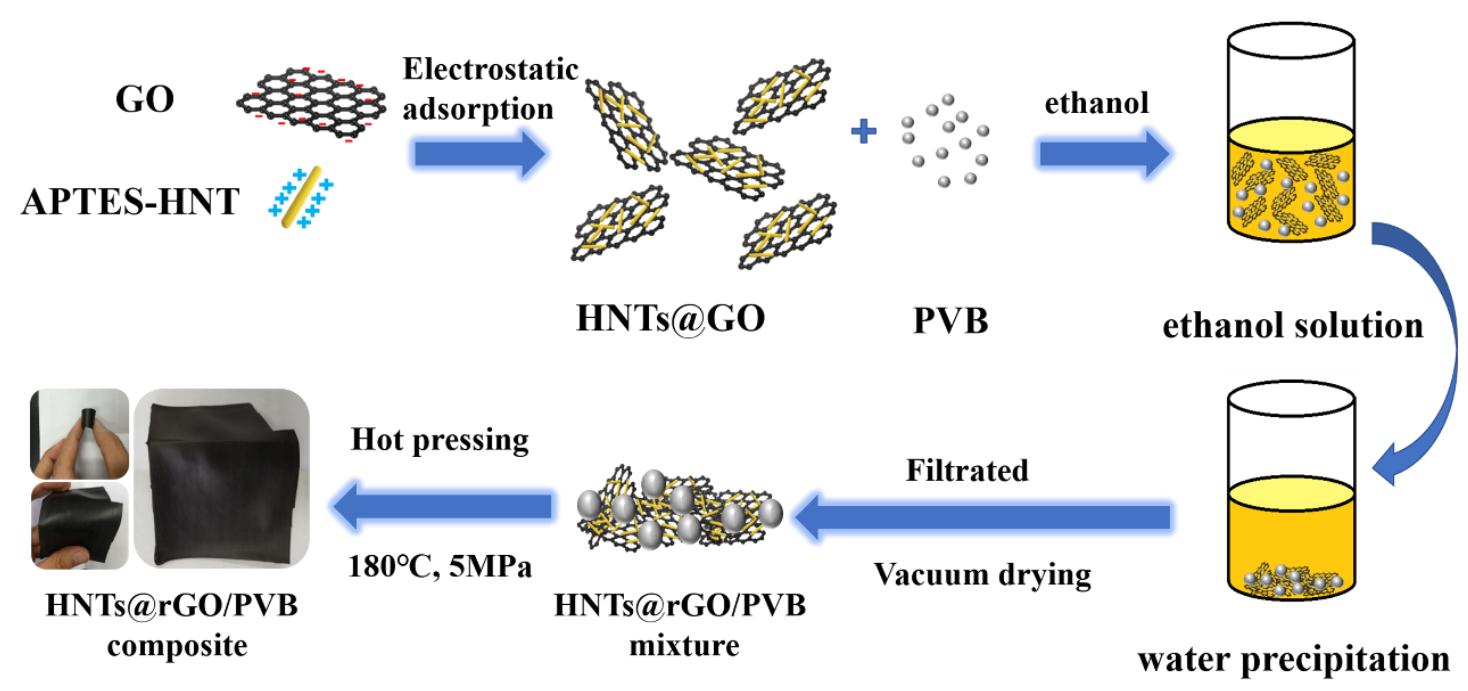

Fig. 1 Mechanism of fabricating HNTs@GO micro-sheet and procedures of preparing HNTs@rGO/PVB composite (digital photos exhibit the 10wt\%HNTs@5wt\%rGO/PVB composite)

\subsection{Characterization}

Fourier transform infrared spectroscopy (FTIR, Bruker Tensor 27, Bruker Daltonics, Germany) was employed to characterize the grafted APTES on the surface of HNTs. X-ray photoelectron spectroscopy (XPS, Thermo ESCALAB 250, TheroFisher Scientific, USA) was used to further confirm the existence of the APTES after the chemical modification of HNTs. Raman spectra (DXR3, Thermo Scientific DXR2, USA) were used to characterize the graphitization degree of GO before and after heat reduction. Zeta potential measurements (Zetasizer 3000, Malvern Instruments, Britain) were performed to verify the charge property of modified HNTs and GO at different $\mathrm{pH}$ values. The crystal structure of the HNTs@GO before and after heat reduction was analyzed by X-ray diffraction (XRD, XRD-7000, Shimadzu, Japan) in the 5-90 range. The microstructure of PVB composites were investigated on a scanning electron microscope instrument (SEM, JSM-7401F, JEOL, Japan) with an acceleration voltage of $5 \mathrm{kV}$. Transmission electron microscope (TEM, Tecnai, model G220, USA) was 
utilized to acquire detailed microscopic morphology of the HNTs@GO micro-sheet. The dielectric properties of the samples were measured on the impedance analyzer (Agilent 4294A, USA) in a frequency range from $40 \mathrm{~Hz}$ to $30 \mathrm{MHz}$. The breakdown strength was tested using a polarization device (HYJH-3/50kV, Dongxiangyu Corporation, China) within a voltage range from 0 to $50 \mathrm{kV}$. Vaseline was painted around the electrode to prevent the flashover puncture.

\section{Results and discussion}

\subsection{The microstructure and morphology of HNTs@GO micro-sheets}

It has been demonstrated that nano or microparticles with abundant hydroxy groups on the surface are easily negatively charged in water circumstances due to proton effect [23]. Besides, the hydroxy groups on the outmost surface can be modified by other chemical substances which is beneficial to further functionalization of nanoparticles. An electrostatic self-assembly method was operated to synthesize the HNTs@GO micro-sheet. The hydroxy-rich HNT nanoparticles were firstly modified by reacting with APTES. When the outmost surface of APTES-HNTs was converted into a positively charged surface under acidic conditions resulting from the existence of amine-tailed short organic chains, the combination with GO could occur driven by the electrostatic force between the negative GO sheets and positive HNTs, thus obtaining the HNTs@GO micro-sheet [24-25]. FTIR spectra of pristine and APTES modified HNTs were shown in Fig. 2a. For unmodified HNTs, the adsorption peaks appeared at high wavenumber region around $3700 \mathrm{~cm}^{-1}$ and $3620 \mathrm{~cm}^{-1}$ related to hydroxy groups 
indicating the existence of interlayer hydrogen bonding on the surface of HNTs [24]. The $1103 \mathrm{~cm}^{-1}, 1307 \mathrm{~cm}^{-1}$ adsorption band corresponding to the stretching vibration of Si-O groups came from silicate in HNTs. After modification, the adsorption peaks appeared at $1489 \mathrm{~cm}^{-1}, 1560 \mathrm{~cm}^{-1}$, corresponded to stretching and bending vibrations of $-\mathrm{NH}_{2}$, and the adsorption peaks around $2940 \mathrm{~cm}^{-1}$ indicated the antisymmetric stretching vibration of -CH2 groups in APTES molecules [26]. To further testify the existence of $-\mathrm{NH}_{2}$ groups, the nitrogen element was analyzed by XPS spectrum (Fig. 2b). The peak located at about $400 \mathrm{eV}$ can be attributed to the nitrogen on $\gamma$-aminopropyl triethoxysilane and the strengthened adsorption peak of oxygen $(530 \mathrm{eV})$ and silicon $(103 \mathrm{eV})$ also demonstrated the successful grafting of Si-O groups [24]. It should be noted that for pristine HNTs, the Si element peak around $100 \mathrm{eV}$ was also prominent in the spectrum because HNTs was a sort of natural ceramic nanotubes which possessed special structures consisting of Gibbsite octahedral sheet (Al-OH) groups on the internal surface and siloxane groups $(\mathrm{Si}-\mathrm{O}-\mathrm{Si})$ on the outer surface. Also, a noteworthy carbon peak appeared at $300 \mathrm{eV}$ corresponding to the $\mathrm{CaCO}_{3}$ structure in HNTs which was significantly different from carbon element from alkyl chains [24]. The prepared APTES-HNTs were easily re-dispersed in the water. By regulating the $\mathrm{pH}$ value of APTES-HNT solution, Zeta potential can be varied in a wide range because of the different protonation degree of hydrogen ion (Fig. 2c). With the zeta potential of the system converting to less than zero, the positively charged HNT surface can be adsorbed on the negatively charged GO surface due to the electrostatic adsorption. XRD 
patterns of GO, rGO, and HNTs@rGO were displayed in Fig. 2d. The peak around $12^{\circ}$ $\mathrm{H}(001)$ and $21^{\circ} \mathrm{H}(110)$ proved the existence of HNTs in HNTs@GO micro-sheet and the weak peaks at $24.3^{\circ}(002)$ and $43.6^{\circ}(100)$ can be attributed to reduction of rGO and the disappearance of the peak at $9.7^{\circ}(001)$ corresponding to GO indicated that the reduction of GO was completed [24].

The morphology of HNTs@GO micro-sheets with different HNTs and GO weight ratio are shown in Fig. 3. The self-assembled HNTs@GO exhibited good integrity, and all HNTs were perfectly adsorbed on GO sheets ((Fig. 3b, c) compared with mixtures consisting of HNTs and GO where some of the HNTs were isolated at the edge of GO micro-sheet (Fig. 3a). A more exquisite structure can be observed from high-resolution SEM in Fig S2. that the HNTs were wrapping tightly by the rGO which indicated there was a good electrostatic interaction between HNTs and rGO in HNTs@GO hybrids. With the increasing of HNT's weight ratio, the dispersion of HNTs on GO sheets became less uniform and serious aggregation can be observed from Fig. 3d. By comprehensively considering the total amount of fillers in the PVB matrix and the coating effect of HNTs on GO, an optimized weight ratio $\mathrm{x} / \mathrm{y}=2 / 1$ was chosen to prepare the composites so that a good mechanical and dielectric properties can be achieved simultaneously. 

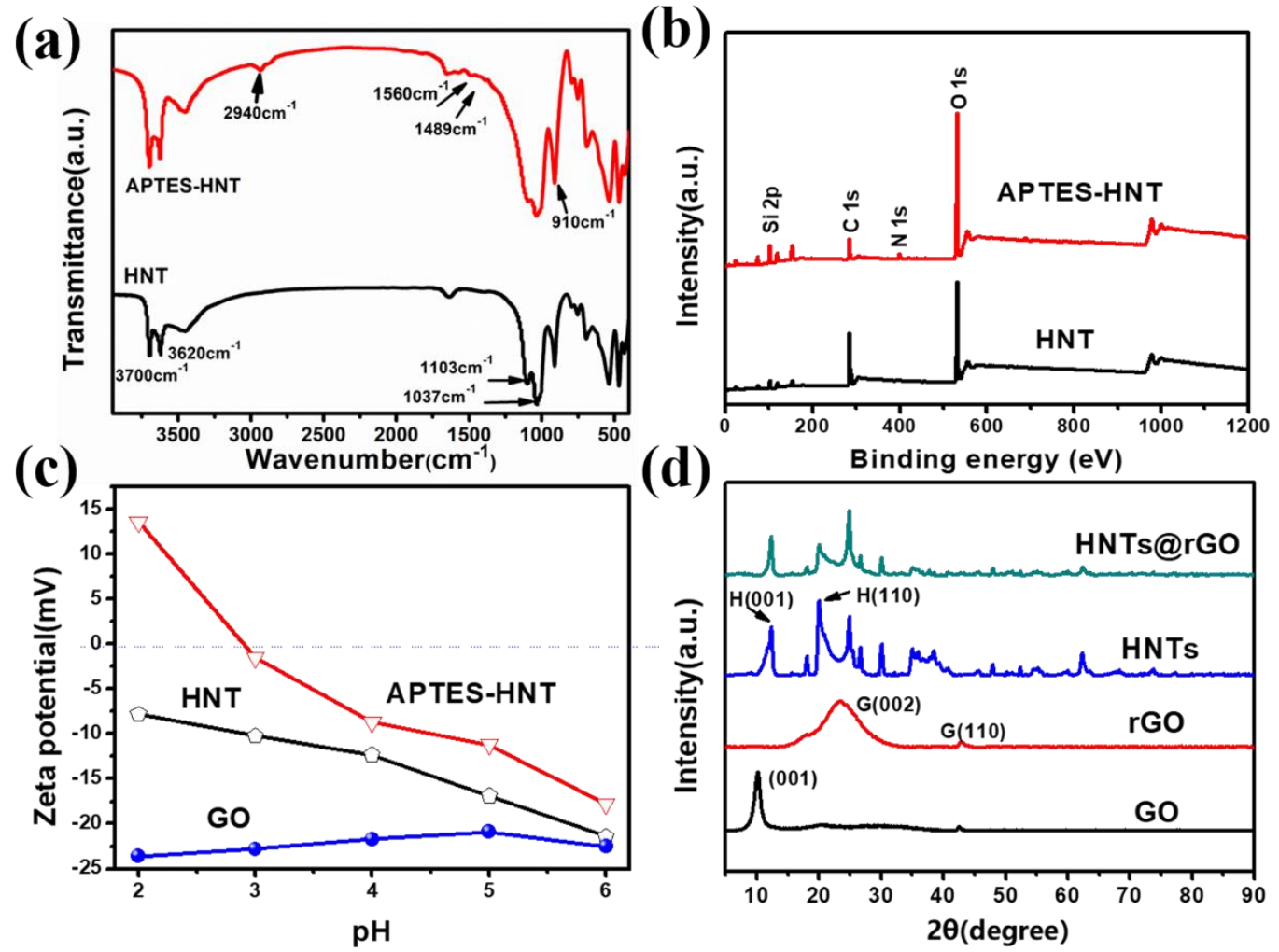

Fig. 2 (a) FTIR and (b) XPS spectra of pristine HNT and APTES-HNTs; (c) Zeta potential of GO, HNTs, and APTES-HNTs in water solvent; (d) XRD patterns of GO, rGO, HNTs and HNTs@ rGO
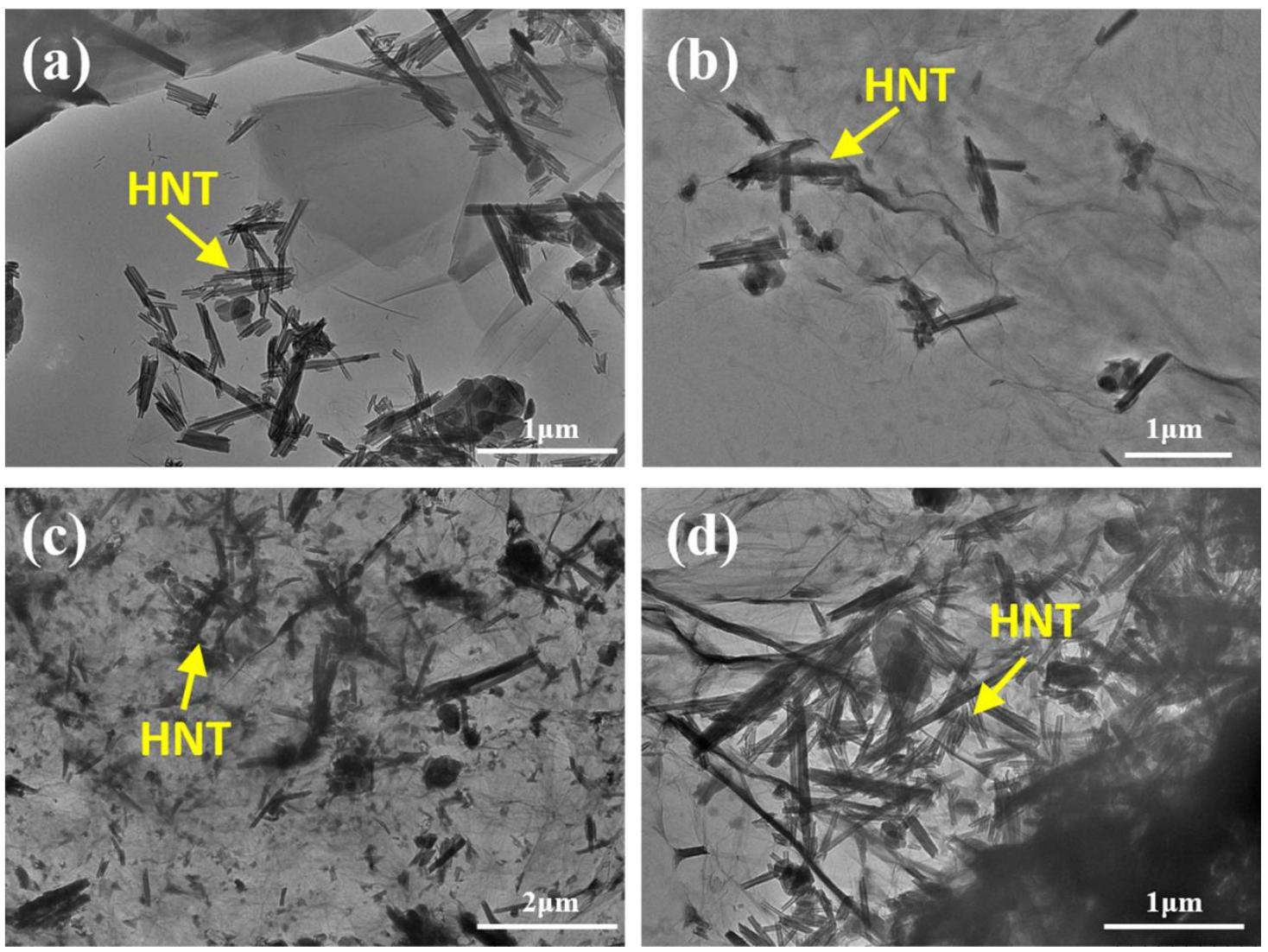
Fig. 3 Morphology of (a) HNTs mixed GO micro-sheets, and self-assembled HNTs@GO micro-sheets with different weight ratio of HNTs and GO: (b) $1: 1$; (c) 2:1; (d) $3: 1$

\subsection{The morphology and dielectric properties of rGO/PVB and}

\section{HNTs/PVB composites}

The morphology of rGO/PVB composites is shown in Fig.4. It can be observed that the low content of rGO fillers showed excellent dispersion state in the PVB matrix. This was mainly attributed to the interaction of affluent carboxyl or hydroxy groups on the surface of GO before the heat reduction process with polar groups in the PVB matrix [27]. With the growth of rGO loadings, the rGO fillers tended to aggregate together and formed thicker sheets (yellow arrow) whereas an excellent compatibility still can be found from the SEM images. These results indicated that GO can be a good candidate to assist the dispersion of other nanoparticles.
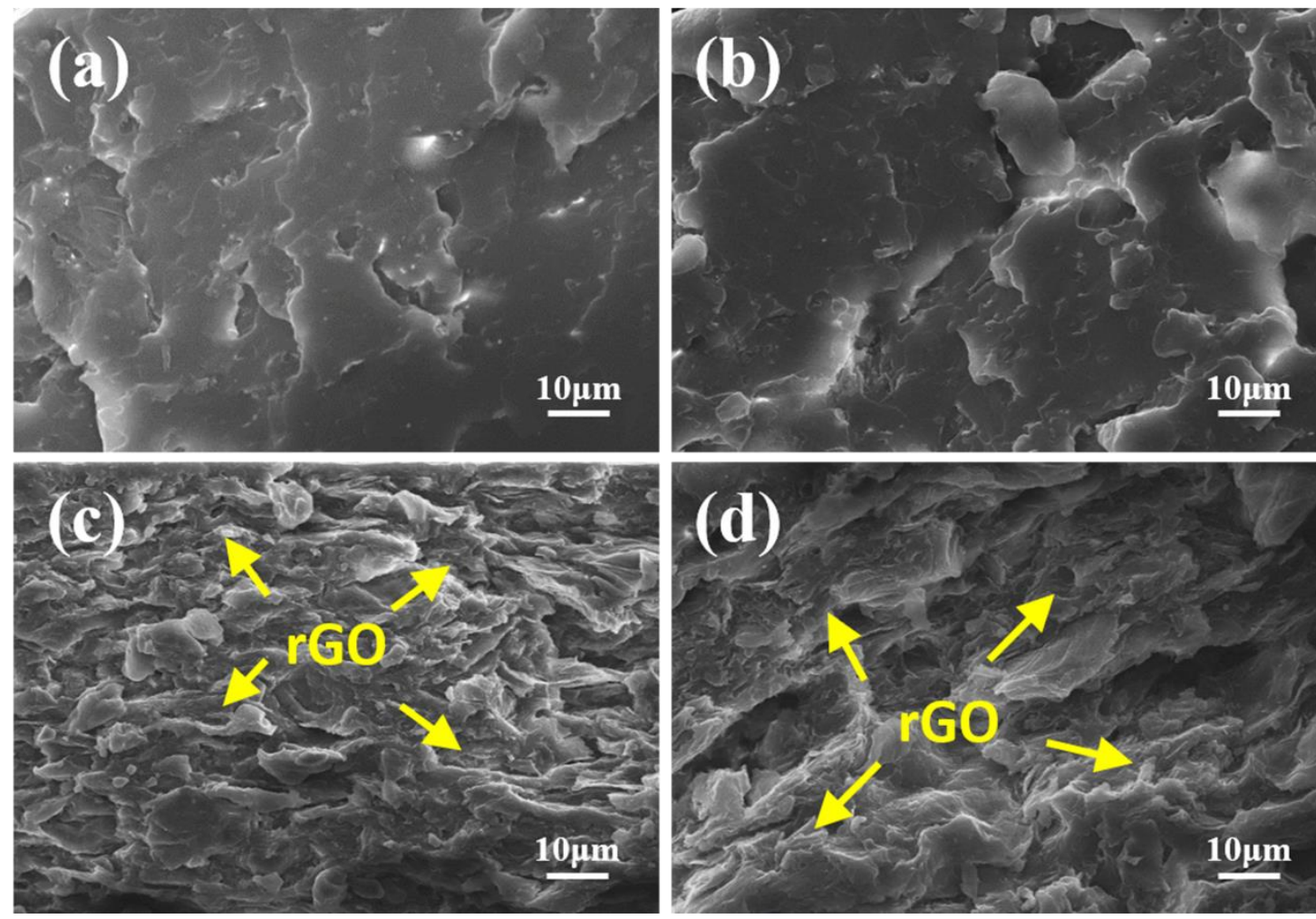

Fig. 4 SEM images of rGO/PVB composites with various filler loadings: (a) $1 \mathrm{wt} \%$ rGO/PVB; (b) 7wt $\%$ rGO/PVB; (c) 15wt\% rGO/PVB; (d) 30wt\% rGO/PVB 
Before analyzing the dielectric properties of HNTs@rGO/PVB composites, two sorts of basic control samples, i.e. rGO/PVB and HNTs/PVB composites, were firstly investigated. Fig.5a and Fig.5b showed the frequency-dependent dielectric constant and dielectric loss of rGO/PVB composites with various rGO loading at room temperature. It can be observed that the dielectric property of rGO/PVB composites with low content (below $7 \mathrm{wt} \%$ ) of rGO was almost independent of frequency in the range of $10^{3}-10^{7} \mathrm{~Hz}$, which was consistent with Maxwell-Wagner-Sillars interfacial polarization as reported in some previous research [28-31]. With the increase of rGO loading, the frequency dependence of the dielectric property gradually became stronger in the range of $10^{3}-10^{7}$ $\mathrm{Hz}$, demonstrating the transition from insulator to semiconductor. Both dielectric constant and dielectric loss increased with the loading of fillers (Fig.5d) at the low frequency $\left(10^{3} \mathrm{~Hz}\right)$. For instance, the dielectric constant of PVB composites containing $1 \mathrm{wt} \% \mathrm{rGO}$ was about 4.7 at frequency of $10^{3} \mathrm{~Hz}$ and then increased to 16.1 after adding $20 \mathrm{wt} \%$ fillers, which was over 5 times higher than that of pure PVB polymer (about 3.2). When the loading of rGO was up to $30 \mathrm{wt} \%$, the dielectric constant exhibited a high value within the low-frequency range $\left(<10^{4} \mathrm{~Hz}\right)$ but a sharp decline at high-frequency range $\left(>10^{4} \mathrm{~Hz}\right)$. These phenomena can be attributed to the leakage current of composites at such a high loading, which also induced high dielectric loss $(>500)$. The conductive rGO and insulating PVB constituted a typical electrical percolation system, in which the percolation threshold $\left(\rho_{c}\right)$ was a crucial parameter representing the connection of conductive fillers in the formation of continuous conducting network 
[32-34]. This system was commonly described by the power-law equation which was shown Eq. (1)

$$
\sigma=\sigma_{0}\left(\rho_{c}-\rho\right)^{-t}, \rho_{c}>\rho
$$

where $\sigma$ was the AC conductivity of the composites and the $\sigma_{0}$ was a constant. $\rho_{\mathrm{c}}$ was the percolation threshold, and $\mathrm{t}$ was the critical conductivity exponent [32].

It can be observed from Fig.5c that AC conductivity of rGO/PVB composites with low filler loadings of $\mathrm{rGO}(<20 \mathrm{wt} \%)$ strongly depended on the frequency and the conductivity value at $10^{3} \mathrm{~Hz}$ of all samples showed a low level $\left(<10^{-6} \mathrm{~S} / \mathrm{cm}\right)$, which proved the insulation and dielectric nature of the materials. As the rGO filler content reached $30 \mathrm{wt} \%$, the conductive networks were formed in the PVB matrix and a stable high value of conductivity can be obtained which was almost independent within $10^{3}-10^{6} \mathrm{~Hz}$. The formation mechanism of rGO conductive networks are shown in Fig. 5e. To calculate $\rho_{\mathrm{c}}$, the log-log curve of $\mathrm{AC}$ conductivity at $1 \mathrm{kHz}$ as a function of $\rho$ was plotted, as shown in Fig. 5e inset. Least-squares linear fitting revealed that the $\rho_{\mathrm{c}}$ of the $\mathrm{rGO} / \mathrm{PVB}$ composites at $1 \mathrm{kHz}$ was $25 \mathrm{wt} \%$ and $\mathrm{t}=3.3$. Such a high $\rho_{\mathrm{c}}$ was attributed to the relatively low conductivity $(1.1 \mathrm{~S} / \mathrm{cm}$, see Fig $\mathrm{S} 2$. b) of rGO sheets under mild heat reduction $\left(180^{\circ} \mathrm{C}, 2 \mathrm{~h}\right)$. 

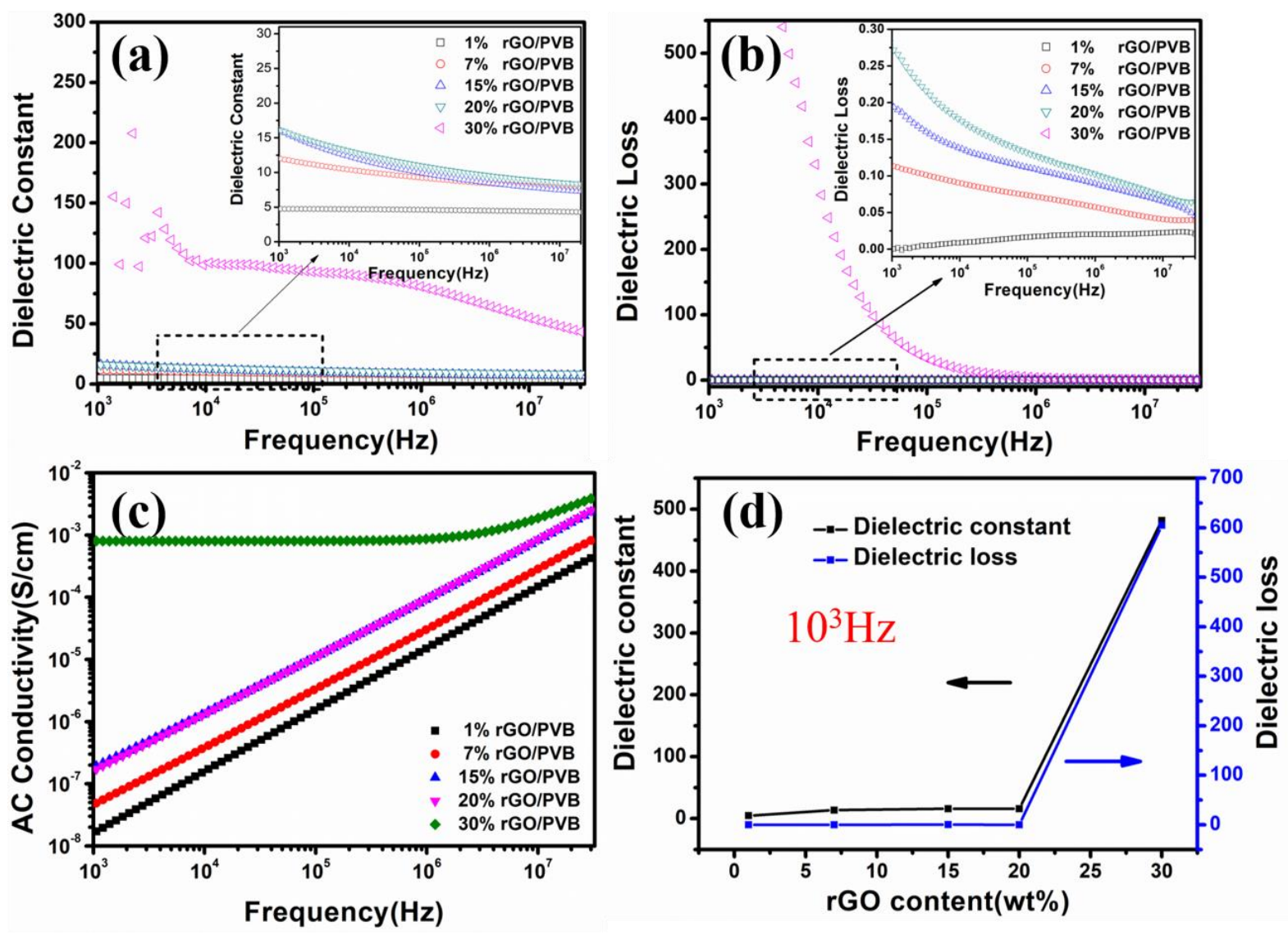

(e)
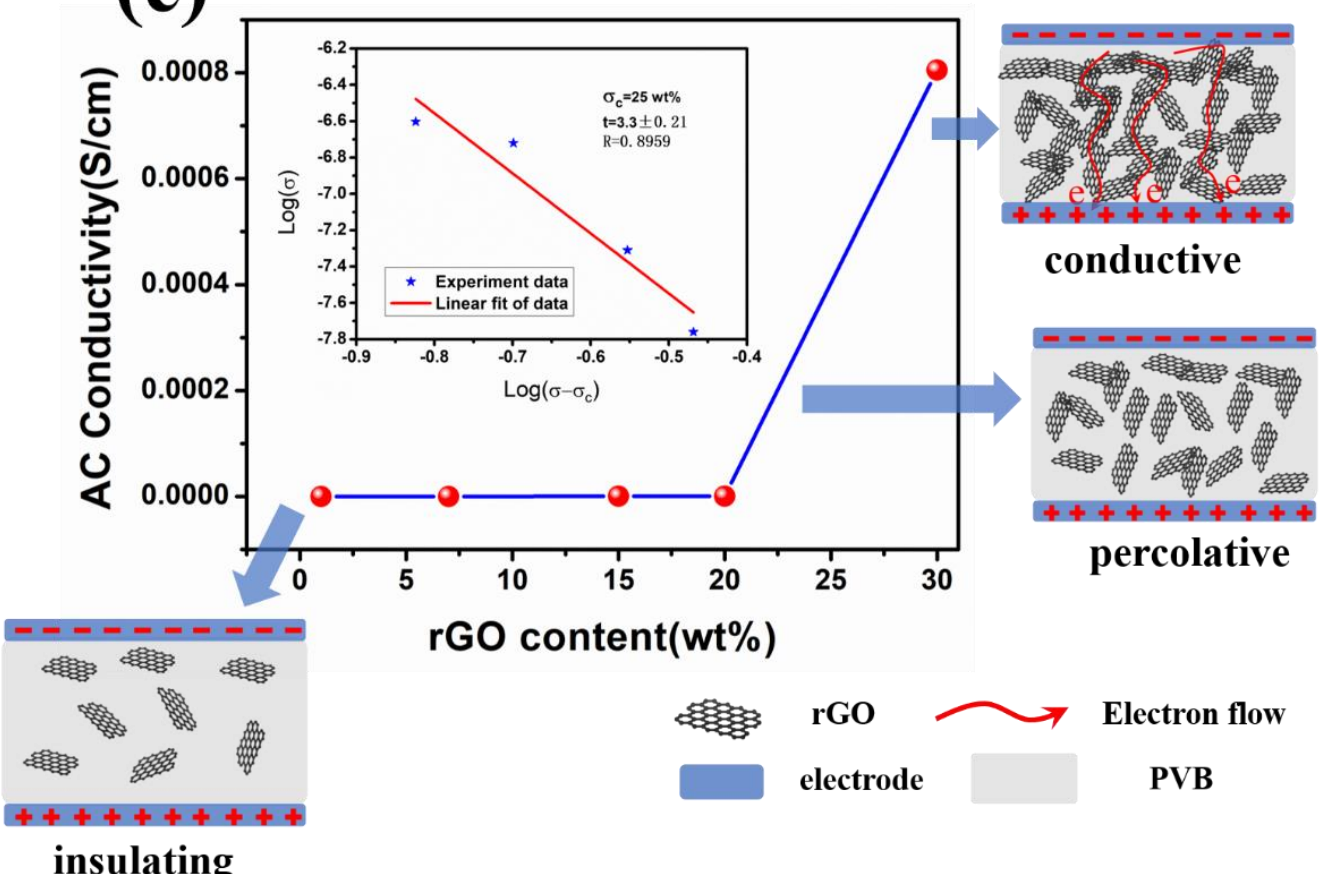

rGO content(wt\%)

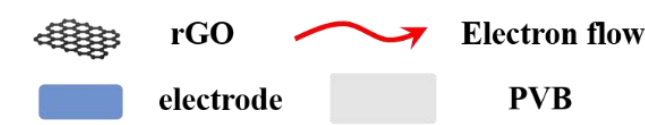

Fig. 5 Dielectric properties of rGO/PVB composites with various rGO loadings: (a) dielectric constant; (b) dielectric loss; (c) AC conductivity; (d) variation trend of dielectric constant and dielectric loss with rGO content at $10^{3} \mathrm{~Hz}$; (e) variation of $\mathrm{AC}$ conductivity with $\mathrm{rGO}$ content at $10^{3} \mathrm{~Hz}$ (inset was percolation 
threshold fitting, cartoons were the rGO distribution in PVB matrix.

Fig. 6 reflected the morphology of cross-sectional fracture surfaces of HNTs/PVB composites. Fig. $6 \mathrm{a}$ and $\mathrm{b}$ revealed that fillers were not well dispersed in the PVB matrix but prone to clustering together because of the lack of active groups on the surface of HNTs. Some of the HNTs were pulled out which can be observed from the magnified images in Fig. $6 \mathrm{c}$ and d, indicating the poor compatibility between HNT fillers and PVB matrix.

The dielectric and electrical properties of pure cold-pressed HNT pellet are shown in Fig. 7. It can be seen from Fig.6 a and b that the dielectric constant and dielectric loss of HNTs were 25 and 0.2 at $10^{3} \mathrm{~Hz}$, respectively, which was comparable to traditional ceramics. There was also a loss peak at $10^{4} \mathrm{~Hz}$ which corresponded to the relaxation of groups in the aluminosilicate structure of HNTs [35]. The AC conductivity of HNT pellet (Fig. 7c and Fig. 7d) indicated that charge transition between HNTs was easier than that between HNTs and PVB matrix but the whole HNTs still presented insulator properties. Fig. 7a and Fig. 7b also illustrated the dependency of dielectric constant and dielectric loss of HNTs/PVB composites on the frequency at room temperature. There was simply a slight dropping trend which can be observed in the test frequency from $10^{3}$ to $10^{7} \mathrm{~Hz}$ even though the filler content reached as high as $30 \mathrm{wt} \%$. With the increasing of HNT loadings, the AC conductivity of PVB composites performed a gradual increment in Fig. $7 d$ but the values were still quite low. This result indicated that the interfacial polarization occurred between HNTs and PVB matrix, and electric charges induced by applied electric field were well restrained at the interface of composites. Due 
to the insulation nature of HNT ceramic, there was no formation of conductive network even after incorporating the high content of HNTs.
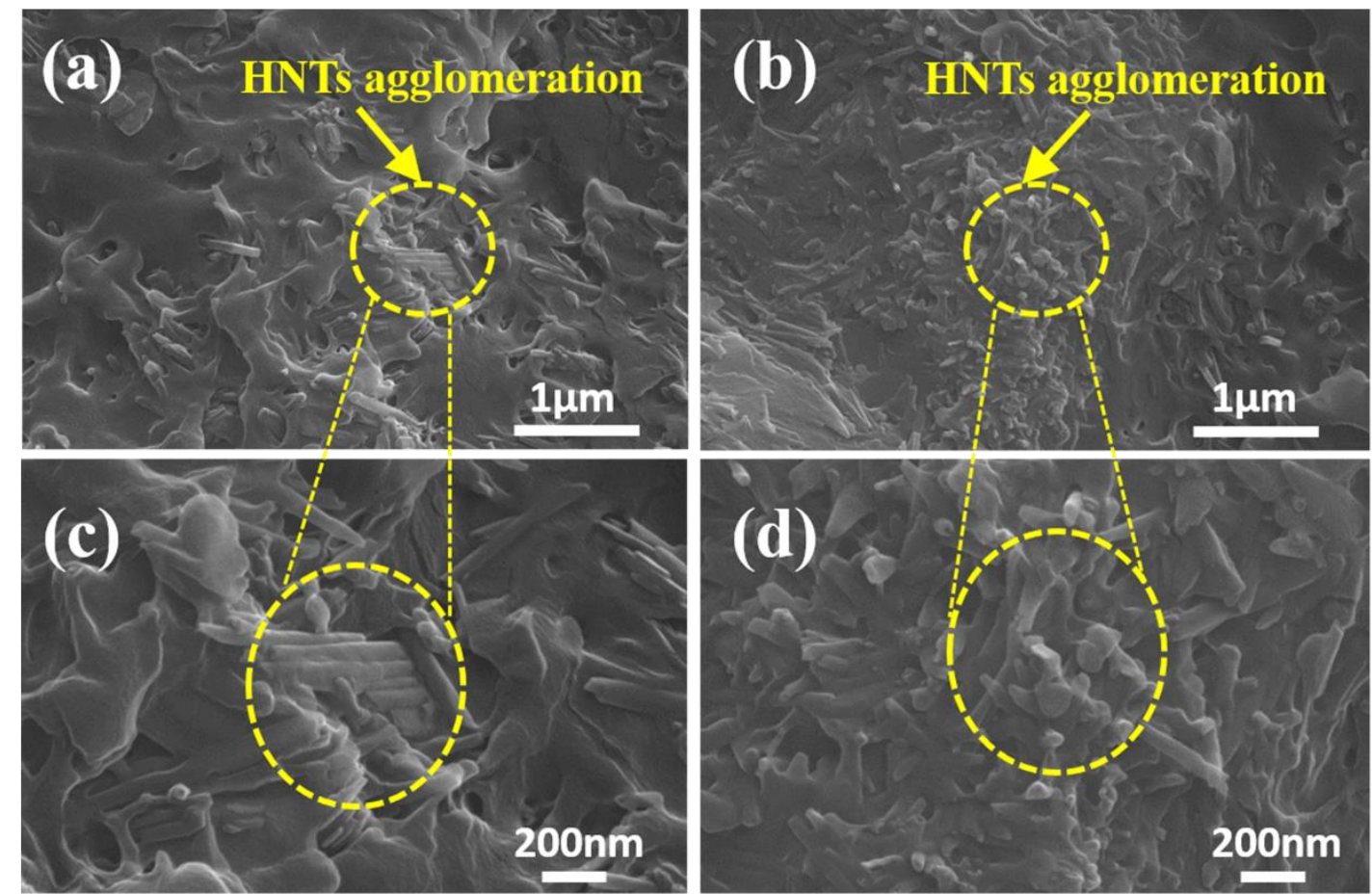

Fig. 6 SEM images of HNTs/PVB composites with various filler loadings: (a) (c) 10wt $\%$ HNTs/PVB; (b) (d) $30 \mathrm{wt} \% \mathrm{HNTs} / \mathrm{PVB}$ 

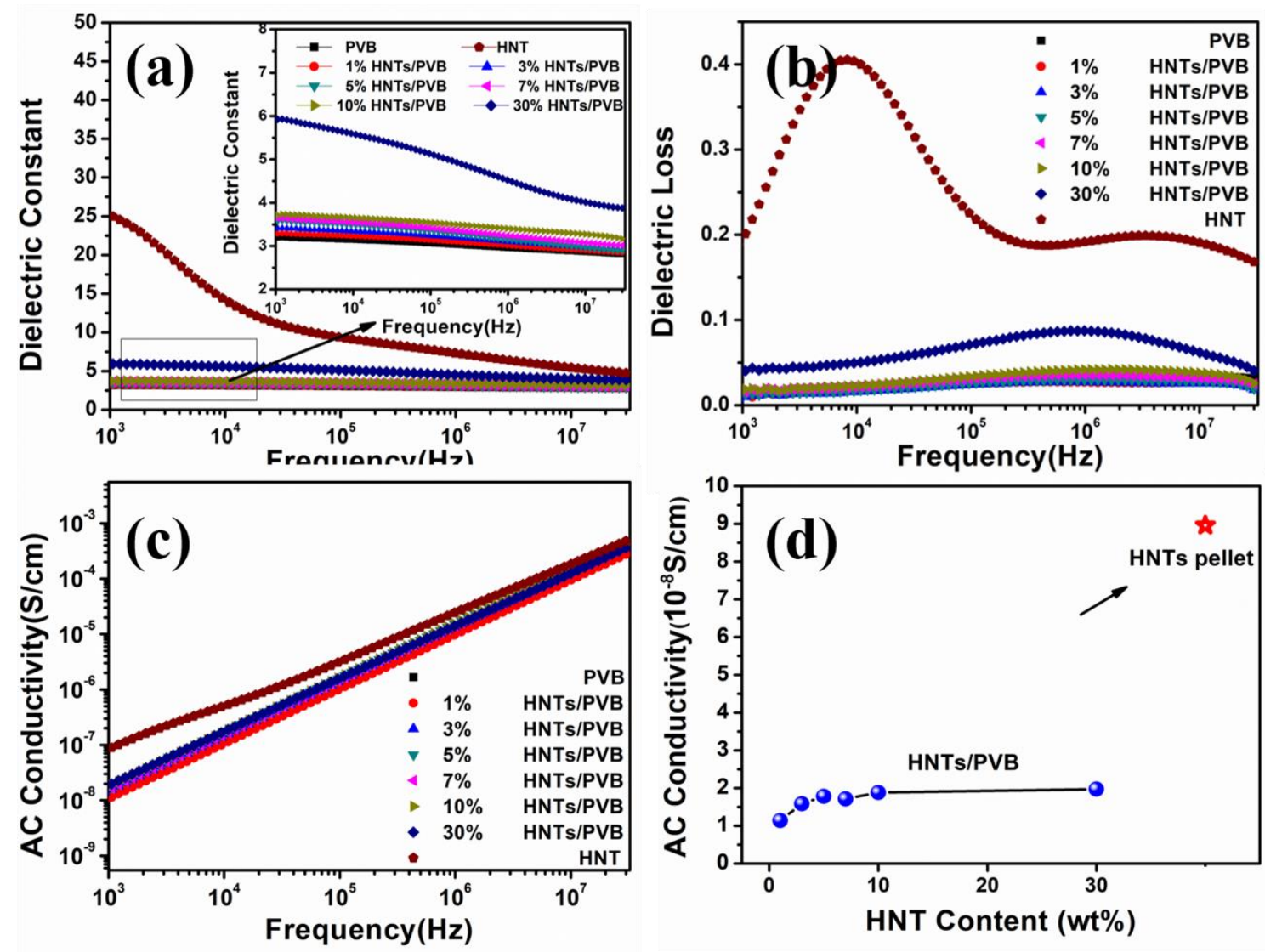

Fig. 7 Dielectric properties of HNTs and HNTs/PVB composites with various filler loadings: (a) dielectric constant; (b) dielectric loss; (c) AC conductivity; (d) AC conductivity of HNTs/PVB and cold-pressed HNTs pellet at $10^{3} \mathrm{~Hz}$

\subsection{The microstructure and dielectric properties of HNTs@rGO/PVB}

\section{composites}

The dielectric enhancement was strongly associated with the microstructure of HNTs@rGO/PVB composites. Fig. 8 presented the cross-sectional fracture surfaces of two sorts of samples with the same $5 \mathrm{wt} \% \mathrm{rGO}$ and $10 \mathrm{wt} \%$ HNTs loading. It can be clearly shown that ordinary mixed HNTs and rGO nanofillers exhibited a very poor dispersion state in the PVB matrix (Fig.8a) compared with that of self-assembled HNTs@rGO (Fig.8b). This fact can be further verified by the elemental mapping (Fig. 8c and d) in which a more uniform Si (the main components of HNTs) element distribution can be observed in a randomly selected visual field. A sketch map 
elucidating the distribution state of HNTs and rGO in two different systems are shown in Fig. 10 e and f. By adsorbing on the surface of rGO, HNTs can play a more significant role in the formation of microcapacitors.
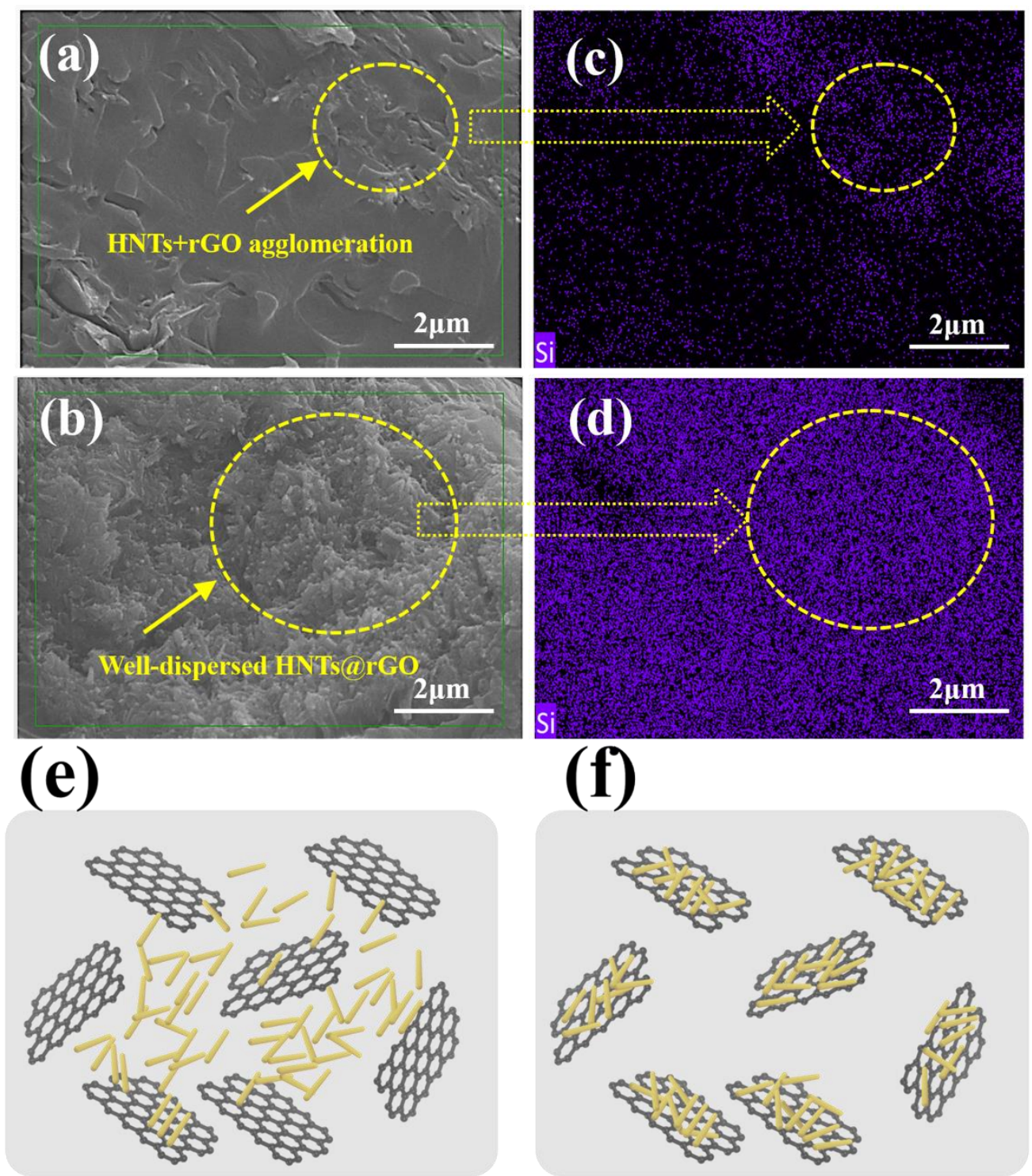

Random not well-dispersed HNTs+rGO

Well-dispersed HNTs@rGO

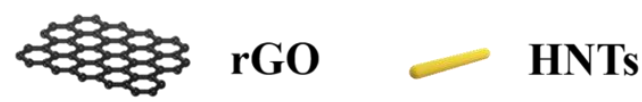

PVB matrix

Fig. 8 SEM images and corresponding Si elemental mapping of (a),(c) 10wt\%HNTs+5wt\%rGO/PVB; (b), (d) 10wt\%HNTs@5wt\%rGO/PVB; (e) and (f) were sketch maps representing ordinary mixing and self-assembly of HNTs and GO, respectively. 
Fig. 9a, b, and d show that the 10wt\%HNTs@5wt\%rGO/PVB composites exhibited very excellent dielectric properties in which the dielectric constant can achieve as high as 150 at $10^{3} \mathrm{~Hz}$, while the dielectric loss was suppressed within 0.12 . It can also be observed in Fig. 9c that the percolation occurred at a very low concentration of rGO fillers ( $>5 \mathrm{wt} \%)$, which was much lower than that of rGO/PVB samples (30wt\%). The microcapacitor model can give a good explanation to such a high dielectric performance when incorporating HNTs@rGO as nanofillers. An illustration of this model is schematically shown in Fig S3. Based on this model, the capacitance value of each microcapacitor was the key factor to influence the resultant dielectric performance. Firstly, the capacitance value was influenced by its effective micro-electrode areas which largely depended on the dispersion state of fillers in the composite. Secondly, the charge storage capacity of the microcapacitor was directly determined by the conductivity of micro-electrode. Thirdly, the dielectric layer between two micro-electrodes must be thin enough so that a giant capacitance can be achieved [36]. Besides, the dielectric layer should prohibit the direct contact of micro-electrode, otherwise, the high dielectric loss would be caused by the formation of conductive networks [37-40].

Fig. 10 presented the possible microcapacitor component in the resulting composite system. In this system, the rGO acted as micro-electrodes with a large sheet size showed good dispersion state in the PVB matrix which has been demonstrated in our previous analysis. For dielectric layers in the middle of two micro-electrodes, they 
can be classified into three types. The first type was the HNTs sandwiched between two adjacent rGO micro-sheets (Fig. 10 I). For such a microcapacitor, HNTs played important roles, not only prevented direct contact between conductive rGO, which was the main source of high dielectric loss but also acted as a thinner high dielectric layer contributing to large capacitance. The second type was the HNTs network between rGO sheets with remote distance (Fig. 10 II), this sort of microcapacitor possessed relatively low capacitance because of its thicker equivalent dielectric layers. The third type was the thin PVB layer in the middle of rGO sheets (Fig. 10 III). Such thin insulation usually cannot completely restrain the charge migration between the rGO because of the tunneling effect [41-42], thus a mild percolation phenomenon can be observed which would lead to a big increase of dielectric constant and slight rise of dielectric loss. It should be noted that the tunneling effect appeared at such a low content of rGO $(>5 \mathrm{wt} \%)$ because of the volume exclusion effect from 1D nanofillers (HNTs) [43-45]. For comparative study, HNTs + rGO/PVB composites were prepared by mixing HNTs and rGO in the PVB matrix. It can be spotted from Fig. 9 (e) and (f) that even though the rGO content reached a very high value of $15 \mathrm{wt} \%$, the percolative phenomenon still did not appear. The dielectric constant of the composite samples with 30wt\% HNTs and $15 \mathrm{wt} \% \mathrm{rGO}$ was merely 5 at $10^{3} \mathrm{~Hz}$. There was no synergistic effect from HNTs and rGO microsheets.

Fig. 10 also provided an assumption on the mechanism of energy conversion inside the HNTs@rGO hybrids based on the microcapacitors theory. For each type of 
microcapacitors, the capacitance was mainly dependent on the thickness of dielectric layers which was described by the Eq. (2)

$$
\mathrm{C}=\mathrm{KS} / \mathrm{L}
$$

where $\mathrm{C}$ was the capacitance of the microcapacitor and $\mathrm{S}$ was electrode area. $\mathrm{K}$ was a constant, and L was the thickness of the dielectric layers [46-47]. Based on the microstructure of the three types of microcapacitors, it can be seen that type (I) and type (III) held a greater energy storage ability so that a larger capacitance can be obtained under external electric field. 

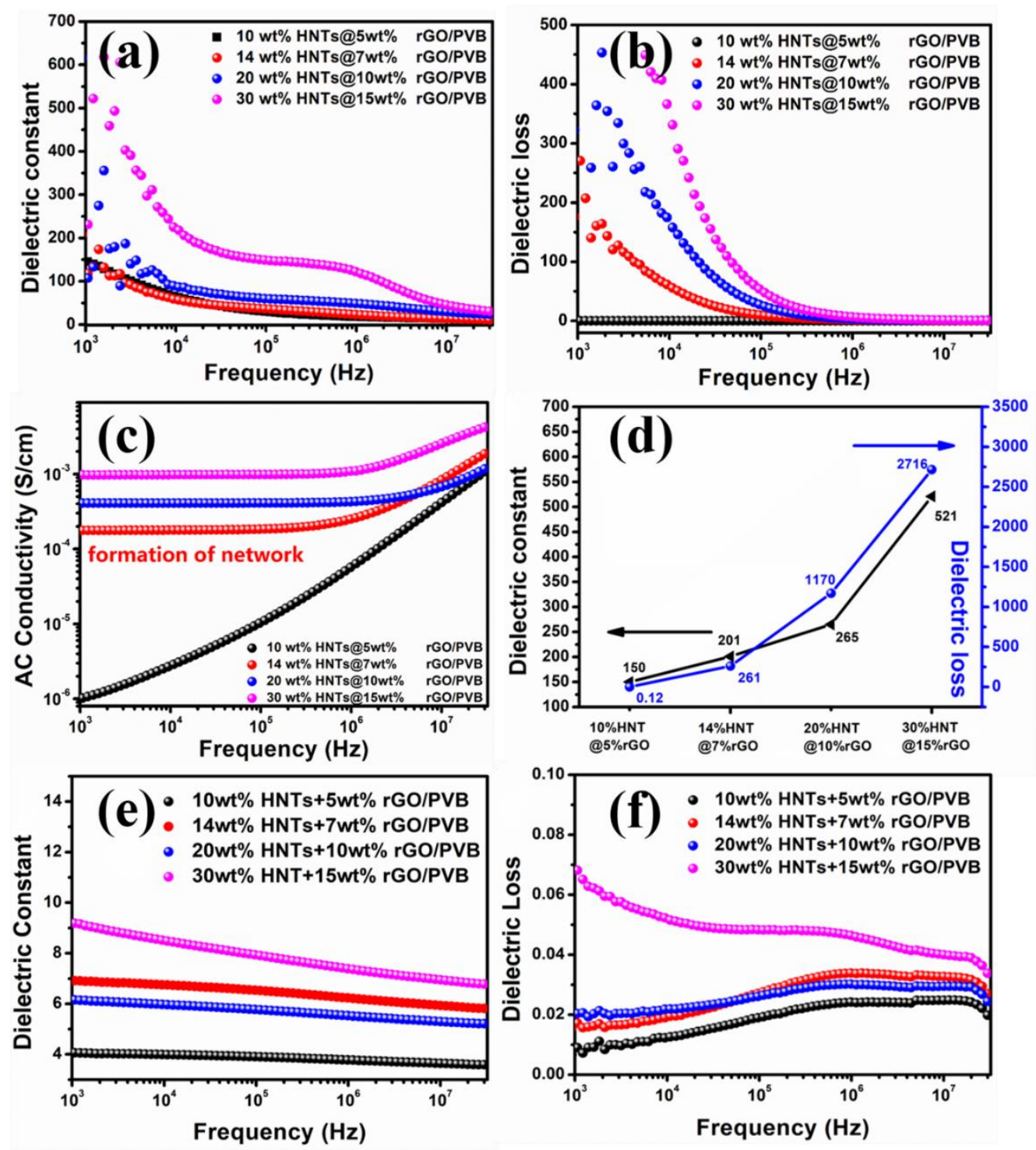

Fig. 9 Dielectric properties of HNTs@rGO/PVB composites with various filler loadings: (a)dielectric constant; (b)dielectric loss; (c) AC conductivity; (d) comprehensive comparison at $10^{3} \mathrm{~Hz}$. Dielectric properties of (HNTs+rGO)/PVB composites: (e) dielectric constant; (f) dielectric loss 


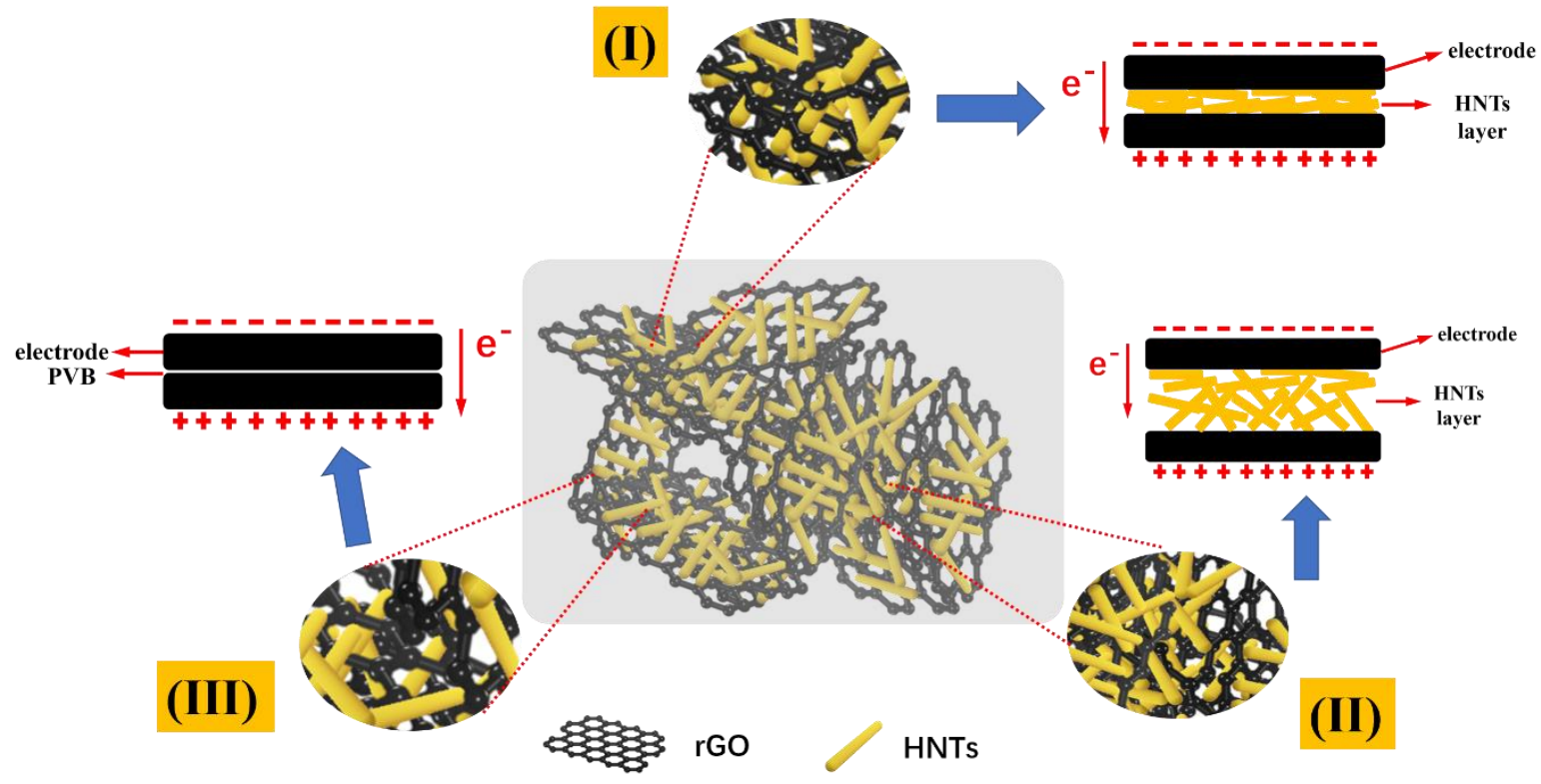

Fig. 10 The synergistic effect of HNTs and rGO on the improvement of dielectric properties in HNTs@rGO composites and energy conversion mechanism in HNTs@rGO hybrids.

\subsection{The breakdown and energy storage properties of HNTs@rGO/PVB composites}

For linear dielectrics, the energy density (Ue) of the material is determined by the breakdown strength (BDS) and dielectric constant described as:

$$
\mathrm{Ue}=1 / 2 \varepsilon_{\mathrm{r}} \varepsilon_{0} \mathrm{E}_{\mathrm{B}}^{2}
$$

where $\varepsilon_{\mathrm{r}}$ and $E_{\mathrm{B}}$ are the relative dielectric permittivity and breakdown strength of the materials respectively. $\varepsilon_{0}$ is the permittivity of vacuum. The conductive fillers/polymer composites are typical linear dielectrics whose energy storage properties are largely dependent on the breakdown strength of the system [48-49]. The Weibull breakdown distribution and Characteristic BDS of the HNTs@rGO/PVB composites with different loading of nanofillers are displayed in Fig. 11. The BDS of HNTs@rGO/PVB composites showed a decreasing trend with increasing loading of HNTs@rGO (Fig. 11b) 
as a result of the structural imperfections caused by the incorporation of nanofillers. By calculating the energy density under breakdown strength according to equation (1), it can be discovered that 10wt\% HNTs@5\%rGO/PVB composites exhibited the most excellent energy storage properties $\left(\mathrm{Ue}=0.8 \mathrm{~J} / \mathrm{cm}^{3}\right)$, which was 13 times higher than that of pure PVB material $\left(\mathrm{Ue}=0.06 \mathrm{~J} / \mathrm{cm}^{3}\right)$. The overall performance of the HNTs@rGO/PVB composites will be well qualified to satisfy the demands in low voltage micro-electronic field where the requirement of breakdown strength is usually no more than $1 \mathrm{kV} / \mathrm{mm}$.

(a)

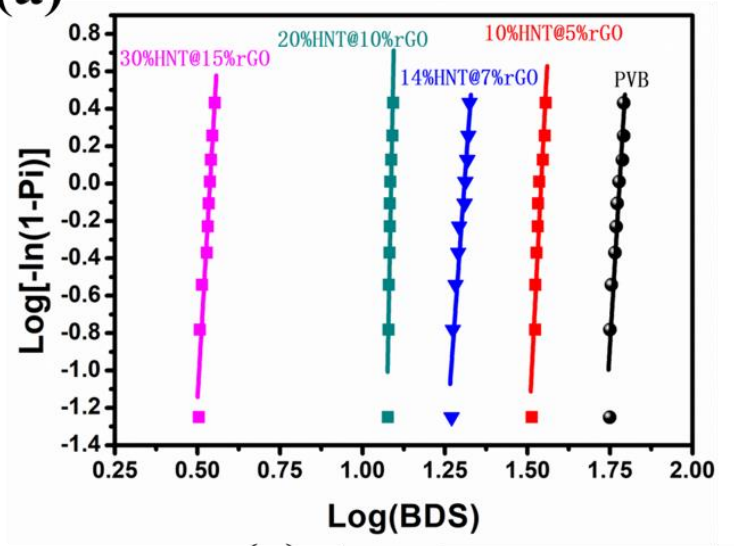

(b)

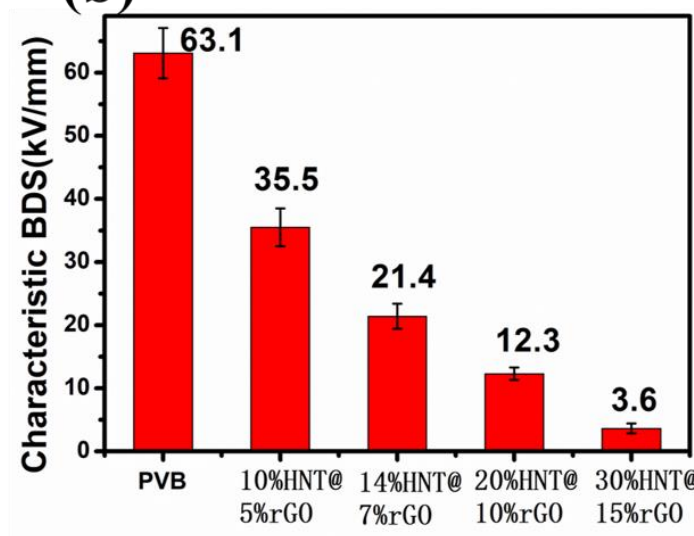

(c)

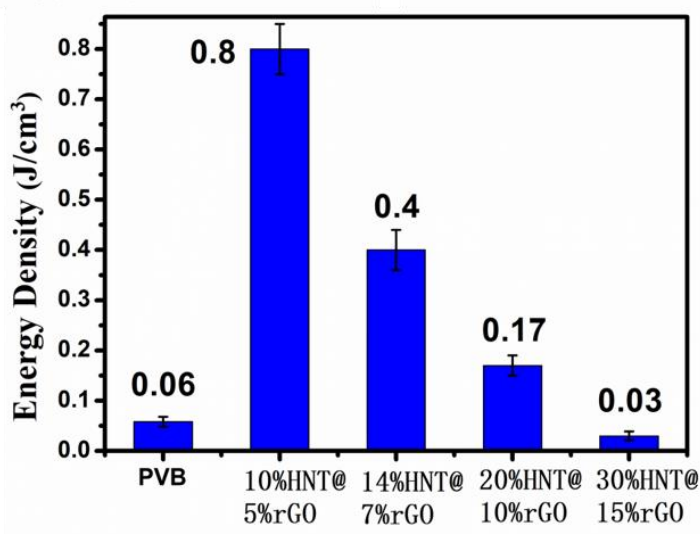

Fig. 11 Breakdown and energy storage properties of HNT@rGO/PVB composites: (a) Weibull distribution; (b) characteristic BDS; (c) energy density

\section{Conclusion}

A flexible HNTs@rGO/PVB functional composites were prepared by using the 
in-situ heat reduction method. The HNTs were adsorbed on the surface of graphene oxide and the micro-sheets exhibited a good integrity. Compared with traditional polymer materials with ceramic coated conducting fillers, the 10wt\%HNTs@5wt\%rGO/PVB composites possessed an ultra-high dielectric constant $\left(\varepsilon^{\prime}\right)$ of $150\left(10^{3} \mathrm{~Hz}\right)$ with a low filling content of $5 \mathrm{wt} \%$ rGO. Furthermore, the dielectric loss of the composites $\left(\tan \delta=0.12,10^{3} \mathrm{~Hz}\right)$ can be controlled in the same magnitude with that of $7 \mathrm{wt} \%$ pure rGO filled PVB $\left(\varepsilon^{\prime}=12.5, \tan \delta=0.1,10^{3} \mathrm{~Hz}\right)$. It was demonstrated that the synergistic effect of rGO and HNTs played an extremely significant role in improving the dielectric constant of the resulting composites and suppressing the dielectric loss. The modification methods and application of semi-conductive nanofillers reported here can be further developed in the fabrication of high-performance dielectric materials.

\section{Acknowledgment}

The authors acknowledge the financial support from the National Natural Science Foundation of China (No. 51873011 and No. U1664251).

\section{Reference}

[1] Huang X, Sun B, Zhu Y, et al. High-k polymer nanocomposites with 1D filler for dielectric and energy storage applications[J]. Progress in Materials Science, 2018.

[2] Zhang X, Jiang J, Shen Z, et al. Polymer nanocomposites with ultrahigh energy density and high discharge efficiency by modulating their nanostructures in three dimensions[J]. Advanced Materials, 2018, 30(16): 1707269.

[3] Li Q, Chen L, Gadinski M R, et al. Flexible high-temperature dielectric materials from polymer nanocomposites[J]. Nature, 2015, 523(7562): 576.

[4] Cao M S, Wang X X, Zhang M, et al. Variable-Temperature Electron Transport and Dipole Polarization Turning Flexible Multifunctional Microsensor beyond Electrical and Optical Energy[J]. Advanced Materials, 2020: 1907156.

[5] Wang X X, Shu J C, Cao W Q, et al. Eco-mimetic nanoarchitecture for green EMI 
shielding[J]. Chemical Engineering Journal, 2019, 369: 1068-1077.

[6] Su Y, Ren Y, Chen G X, et al. Synthesis of high-k and low dielectric loss polymeric composites from crosslinked divinylbenzene coated carbon nanotubes[J]. Polymer, 2016, 100: 179-187.

[7] Balci O, Polat E O, Kakenov N, et al. Graphene-enabled electrically switchable radar-absorbing surfaces[J]. Nature communications, 2015, 6(1): 1-10.

[8] Li B, Sui G, Zhong W H. Single negative metamaterials in unstructured polymer nanocomposites toward selectable and controllable negative permittivity[J]. Advanced Materials, 2009, 21(41): 4176-4180.

[9] Shu J C, Cao M S, Zhang M, et al. Molecular Patching Engineering to Drive Energy Conversion as Efficient and Environment-Friendly Cell toward Wireless Power Transmission[J]. Advanced Functional Materials, 2020: 1908299.

[10] Li W, Song Z, Qian J, et al. Enhancing conjugation degree and interfacial interactions to enhance dielectric properties of noncovalent functionalized graphene/poly (vinylidene fluoride) composites[J]. Carbon, 2019, 141: 728-738.

[11] Li Y, Shi Y, Cai F, et al. Graphene sheets segregated by barium titanate for polyvinylidene fluoride composites with high dielectric constant and ultralow loss tangent[J]. Composites Part A: Applied Science and Manufacturing, 2015, 78: 318-326.

[12] Wan Y J, Zhu P L, Yu S H, et al. Barium titanate coated and thermally reduced graphene oxide towards high dielectric constant and low loss of polymeric composites[J]. Composites Science and Technology, 2017, 141: 48-55.

[13] Li Y, Fan M, Wu K, et al. Polydopamine coating layer on graphene for suppressing loss tangent and enhancing dielectric constant of poly (vinylidene fluoride)/graphene composites[J]. Composites Part A: Applied Science and Manufacturing, 2015, 73: 85-92.

[14] Fleming M S, Mandal T K, Walt D R. Nanosphere-microsphere assembly: methods for core-shell materials preparation[J]. Chemistry of materials, 2001, 13(6): 2210-2216.

[15] Wu C, Huang X, Wu X, et al. TiO2-nanorod decorated carbon nanotubes for high-permittivity and low-dielectric-loss polystyrene composites[J]. Composites Science and Technology, 2012, 72(4): 521-527.

[16] Dang Z M, Wang L, Yin Y I, et al. Giant dielectric permittivities in functionalized carbon-nanotube/electroactive- ${ }^{-}$polymer nanocomposites[J]. Advanced Materials, 2007, 19(6): 852-857.

[17] Yuan J K, Li W L, Yao S H, et al. High dielectric permittivity and low percolation threshold in polymer composites based on $\mathrm{SiC}$-carbon nanotubes micro/nano hybrid[J]. Applied physics letters, 2011, 98(3): 032901.

[18] Rogti F, Ferhat M. Maxwell-Wagner polarization and interfacial charge at the multi-layers of thermoplastic polymers[J]. Journal of Electrostatics, 2014, 72(1): 91-97.

[19] Dong L, Xiong C, Quan H, et al. Polyvinyl-butyral/lead zirconate titanates composites with high dielectric constant and low dielectric loss[J]. Scripta materialia, 2006, 55(9): 835-837.

[20] Ismail H, Salleh S Z, Ahmad Z. Curing characteristics, mechanical, thermal, and 
morphological properties of halloysite nanotubes (HNTs)-filled natural rubber nanocomposites[J]. Polymer-Plastics Technology and Engineering, 2011, 50(7): 681-688.

[21] Wang Z, Xu D, Huang Y, et al. Facile, mild and fast thermal-decomposition reduction of graphene oxide in air and its application in high-performance lithium batteries[J]. chemical communications, 2012, 48(7): 976-978.

[22] Pei S, Cheng H M. The reduction of graphene oxide[J]. Carbon, 2012, 50(9): 3210-3228.

[23] Kresge A J, Mylonakis S G, Sato Y, et al. Aromatic protonation. IX. Kinetic protonation of hydroxy-and alkoxybenzenes[J]. Journal of the American Chemical Society, 1971, 93(23): 6181-6188.

[24] Liu Y, Jiang X, Li B, et al. Halloysite nanotubes@ reduced graphene oxide composite for removal of dyes from water and as supercapacitors[J]. Journal of Materials Chemistry A, 2014, 2(12): 4264-4269.

[25] Wu C, Huang X, Wu X, et al. Graphene oxide-encapsulated carbon nanotube hybrids for high dielectric performance nanocomposites with enhanced energy storage density[J]. Nanoscale, 2013, 5(9): 3847-3855.

[26] Majoul N, Aouida S, Bessaïs B. Progress of porous silicon APTES-functionalization by FTIR investigations[J]. Applied Surface Science, 2015, 331: 388-391.

[27] Wei T, Luo G, Fan Z, et al. Preparation of graphene nanosheet/polymer composites using in situ reduction-extractive dispersion[J]. Carbon, 2009, 47(9): 2296-2299.

[28] Li W, Song Z, Qian J, et al. Enhancing conjugation degree and interfacial interactions to enhance dielectric properties of noncovalent functionalized graphene/poly (vinylidene fluoride) composites[J]. Carbon, 2019, 141: 728-738.

[29] McKenzie R, Zurawsky W, Mijovic J. A molecular interpretation of Maxwell-Wagner-Sillars processes[J]. Journal of Non-Crystalline Solids, 2014, 406: 11-21.

[30] Wan Y J, Yang W H, Yu S H, et al. Covalent polymer functionalization of graphene for improved dielectric properties and thermal stability of epoxy composites[J]. Composites Science and Technology, 2016, 122: 27-35.

[31] Wan X, Zhan Y, Zeng G, et al. Nitrile functionalized halloysite nanotubes/poly (arylene ether nitrile) nanocomposites: Interface control, characterization, and improved properties[J]. Applied Surface Science, 2017, 393: 1-10.

[32] Stauffer D, Aharony A. Introduction to percolation theory[M]. CRC press, 2018.

[33] Cao M, Wang X, Cao W, et al. Thermally driven transport and relaxation switching self-powered electromagnetic energy conversion[J]. Small, 2018, 14(29): 1800987.

[34] Cao W Q, Wang X X, Yuan J, et al. Temperature dependent microwave absorption of ultrathin graphene composites[J]. Journal of Materials Chemistry C, 2015, 3(38): 10017-10022.

[35] Cavallaro G, Donato D I, Lazzara G, et al. Films of halloysite nanotubes sandwiched between two layers of biopolymer: from the morphology to the dielectric, thermal, transparency, and wettability properties[J]. The Journal of Physical Chemistry 
C, 2011, 115(42): 20491-20498.

[36] Butler J W, Concordia C. Analysis of series capacitor application problems[J]. Electrical Engineering, 1937, 56(8): 975-988.

[37] Perrier G, Bergeret A. Maxwell-Wagner-Sillars relaxations in polystyrene-glass bead composites [J]. Journal of applied physics, 1995, 77(6): 2651-2658.

[38] Tonkoshkur A S, Glot A B, Ivanchenko A V. Basic models in dielectric spectroscopy of heterogeneous materials with semiconductor inclusions[J]. Multidiscipline Modeling in Materials and Structures, 2017, 13(1): 36-57

[39] Lestriez B, Maazouz A, Gerard J F, et al. Is the Maxwell-Sillars-Wagner model reliable for describing the dielectric properties of a core-shell particle-epoxy system? [J]. Polymer, 1998, 39(26): 6733-6742.

[40] Pecharromán C, Moya J S. Experimental evidence of a giant capacitance in insulator-conductor composites at the percolation threshold[J]. Advanced Materials, 2000, 12(4): 294-297.

[41] Elshurafa A M, Almadhoun M N, Salama K N, et al. Microscale electrostatic fractional capacitors using reduced graphene oxide percolated polymer composites[J]. Applied Physics Letters, 2013, 102(23): 232901.

[42] Nan C W, Shen Y, Ma J. Physical properties of composites near percolation[J]. Annual Review of Materials Research, 2010, 40: 131-151.

[43] Chen Z, Liu Y, Fang L, et al. Role of reduced graphene oxide in dielectric enhancement of ferroelectric polymers composites[J]. Applied Surface Science, 2019, 470: 348-359.

[44] Rahaman M, Chaki T K, Khastgir D. Consideration of interface polarization in the modeling of dielectric property for ethylene vinyl acetate (EVA)/polyaniline conductive composites prepared through in-situ polymerization of aniline in EVA matrix[J]. European Polymer Journal, 2012, 48(7): 1241-1248.

[45] Efros A L, Shklovskii B I. Critical behaviour of conductivity and dielectric constant near the metal - non - metal transition threshold[J]. physica status solidi (b), 1976, 76(2): 475-485.

[46] Bergman D J, Imry Y. Critical behavior of the complex dielectric constant near the percolation threshold of a heterogeneous material[J]. Physical Review Letters, 1977, 39(19): 1222.

[47] Castner T G, Lee N K, Cieloszyk G S, et al. Dielectric anomaly and the metal-insulator transition in n-type silicon[J]. Physical Review Letters, 1975, 34(26): 1627.

[48] Barber P, Balasubramanian S, Anguchamy Y, et al. Polymer composite and nanocomposite dielectric materials for pulse power energy storage [J]. Materials, 2009, 2(4): 1697-1733.

[49] Sun Lili, Zhong Yanli, Crystallization and Dielectric Properties of Carbon Nanofiber/High-density Polyethylene Composites[J]. Journal of Materials Engineering. 2013, 4, 17-22 